\title{
Stimulated Emission Depletion Microscopy Resolves Nanoparticle Assembly on a Porous Membrane Surface
}

Bhanu Bhakta Neupane ${ }^{1,2 \#}$

${ }^{1}$ Center for Analytical Sciences, Kathmandu Institute of Applied Sciences, Kathmandu, Nepal

${ }^{2}$ Department of Chemistry, Amrit Campus, Tribhuvan University, Kathmandu, Nepal

\section{KEYWORDS}

Nanoparticles; self assembly; microscopy; stimulated emission deletion

\section{"Correspondence}

Tel: +977 16924204

E-mail: bneupane@kias.org.np

Article History

Received 29 September 2015

Revised o1 August 2016

Accepted 07 August 2016

\section{Academic Editor}

Suresh Kumar Dhungel

\section{Introduction}

Resolution of an imaging instrument is a measure of its ability to distinguish two closely spaced objects. If we say resolution of an imaging system (D) is $50 \mathrm{~nm}$ then it implies that two objects that are located at distance of $50 \mathrm{~nm}$ can be distinguished as separate objects. In other words, resolution measures detail and clarity in an image. To get a good quality image, both resolution and magnification has to go in parallel. Magnification is the measure of zooming (ratio of physical size of image and object). If a system has low resolution but high zoom then image becomes obscured.

Several types of imaging systems are in use which can be classified as optical, electron microscopic, and scanning probe techniques. In optical technique, an object is illuminated with a light source and any type of signal (scattering, fluorescence, transmitted, etc.) coming from the object is collected. Optical technique of imaging is classified further into nearfield and far-field. In near-field like Near-field Scanning Optical Microscopy (NSOM), sample is illuminated (or signal collected) at immediate vicinity (distance equal to fraction of light wavelength; that is why called near-field) by a sharp metallic tip or aperture having size of 10-50 nm. In far-field, sample is illuminated or collected with a help of optical lens at a distance much greater than the wavelength of light (thus called far-field). Lateral (-XY) resolution (D) of a far-field microscopic system is given by (Stender et al. 2013) (Müller 2006):

$$
D=\frac{\lambda}{2 \times N A}=\frac{\lambda}{2 \times n \sin \theta}
$$

Where, $\lambda$ is wavelength of light used, $n$ is refractive index of intervening medium (i.e. medium between lens and sample which can be air or oil), $\theta$ is half the light collection angle of an objective, and NA equal to $n \sin \theta$ is numerical aperture of the objective. For small $\theta, \sin \theta$ is roughly given by ratio of radius of lens ( $r$ ) to focal length (f) i.e. $\sin \theta=r / f$. Lens having large $r / f$ gives smaller $D$. The reason SLR and DSLR camera incorporate larger diameter lens is because it gives larger $r / f$, which can give better image due to two reasons. Firstly, larger NA means smaller D which helps to give better quality image. Secondly, light collection efficiency scales as (NA) ${ }^{2}$. It means, lens having NA of 2 collects four times more light than I NA lens. Thus, high NA is advantageous in taking image of a dim object. To get small $\mathrm{D}$ (high resolution), according to equation (1), $\lambda$ should be small and NA should be large. It means blue light ( $\lambda$ around $450 \mathrm{~nm}$ ) gives better resolution (smaller $\mathrm{D}$ ) than red light ( $\lambda$ around $700 \mathrm{~nm}$ ). The highest numerical aperture of microscope objective available in market is in the range of 1.3-1.4 (cost of such objective is around $\$ 10,000$ !). If blue light having wavelength of $450 \mathrm{~nm}$ with $1.4 \mathrm{NA}$ objective is used, equation (1) gives theoretical resolution (i.e. D) of 160.7 $\mathrm{nm}$. Above equation is for ideal case which assumes absence of any optical aberrations like astigmatism, coma, refractive index mismatch, etc. Objective having $100 \%$ aberration free do not exist, so, in real experiment, resolution is worse than $160.7 \mathrm{~nm}$ (experimental resolution in the range of $200-250$ $\mathrm{nm}$ is commonly measured). The diameter of light spot that can be formed in the sample (focal plane) is roughly $2 \times \mathrm{D}$. It means, if light spot in focal plane is small resolution becomes high. But because of diffraction phenomenon of light, spot size smaller than $2 \times \mathrm{D}$ is impossible. In this regard, resolution of far field optical microscope is called diffraction limited i.e. diffraction sets limit to the resolution. One has to keep in mind is that even if resolution is $200-250 \mathrm{~nm}$, far-field optical microscopy can see objects smaller than resolution. However, size of the image spot (obtained after measuring scattering or fluorescence or transmitted signal coming from the object) 
becomes bigger, which is close to diameter of light spot in focal plane (i.e. 400-500 $\mathrm{nm}$ in diameter). For example, if image of a nanoparticle having diameter of $50 \mathrm{~nm}$ is measured, because of diffraction limited resolution of 200-250 nm, the image size of this nanoparticle will appear in the range of 400-500 $\mathrm{nm}$ (equal to size of light spot in focal plane). It means that any object having size smaller than light spot will appear as big as light spot. This is something like writing letters with a pen. If tip of the pen (analogous to size of light spot) is big, writing letter smaller than size of tip is impossible.

In near-field optical technique, where sample is illuminated or signal collected at very close distance using tip or pin hole of size in the range of 10-50 nm, light having different property (evanescent electric field component of light) is collected. Such light component is free of diffraction. That way, depending on size of the tip or aperture used and distance, resolution in the range of $20-50 \mathrm{~nm}$ (much smaller $\mathrm{D}$ than predicted by equation 1) can be obtained.

In electron microscopic techniques [like Scanning Electron Microscopy (SEM) and Transmission Electron Microscopy (TEM)] electron beam, instead of light, is used to image a sample. The electrons are accelerated at very high speed using electric field and focused to sample using electro-magnet instead of optical lens. The wavelength $(\lambda)$ of accelerated electron can be calculated from de Broglie equation:

$$
\lambda=\frac{h}{p}=\frac{h}{\sqrt{2 m e V}}
$$

Where, $\mathrm{h}$ is Planck's constant, $\mathrm{p}$ is momentum of electron, $\mathrm{m}$ is mass of electron, $\mathrm{e}$ is electronic charge, and $\mathrm{V}$ is applied voltage. If electrons are accelerated with very high kinetic energy (K.E $=\mathrm{eV})$ of around 100 kilo electron-volts, then from equation (2) $\lambda$ will be in around $0.37 \mathrm{~nm}$. Plugging this $\lambda$ in equation (1), (assuming NA of 1 ) resolution (i.e. D) of around $0.2 \mathrm{~nm}$ is obtained. This resolution is very high (high resolution means smaller $\mathrm{D}$ ) as compared to resolution of 200-250 nm observed in far-field optical microscopy.

In scanning probe microscopies [like Atomic Force Microscopy (AFM) and Scanning Tunneling Microscopy (STM)], a sharp tip having few atoms at the tip curvature is scanned very close to the sample (depending on the mode of operation distance between tip and sample can range from fraction of a $\mathrm{nm}$ to $100 \mathrm{~nm}$ ) and force (in AFM) or tunneling current (in STM) existing between tip and sample is measured. The measurement of such parameters gives image of a sample. Such approaches, like electron microscopic techniques, give sub-nanometer resolution.

Each microscopic technique has its own merits and demerits. Electron microscopic techniques offer excellent resolution but requires sample to be conductive, thin, and dry. In situ techniques however, don't need sample to be perfectly dry. If one likes to image a cell specimen in fixed or live form then electron microscopy does not work. Scanning probe microscopy can image conductive and/or non-conductive samples but requirement of very small sample probe distance limits their application in thick watery biological sample. Near-field optical techniques also demand very small sample to probe distance so their application in thick sample is limited. Far field optical microscopy works well for watery and thick sample but it has limited resolution.

For almost 150 years, after the discovery of diffraction of light, people thought that resolution of far-field optical microscope cannot be better than as predicted from equation (1). In recent years, such limit is broken by engineering the illumination light spot or engineering optical properties of fluorophore (a dye or molecule that gives visible color fluorescence). Using such approaches, resolution of far filed optical microscopy can be increased from $250 \mathrm{~nm}$ to the range of 20-50 nm even in thick watery biological sample. Far filed optical microscope having resolution better than diffraction limited resolution is called optical nanoscope and the microscopy is called as optical nanoscopy. Stimulated Emission Depletion (STED) microscopy is a type of nanoscopy pioneered by Stafen Hell-one of the recipients of 2014 Nobel Prize in Chemistry- in 1994. In STED microscope, a donut shaped laser beam (laser light having dark hole in the centre i.e. virtually no light in the centre) is overlapped with a Gaussian shaped excitation beam. When these beams are perfectly overlapped in -XYZ directions in focal plane of objective, the dyes or fluorophores that are excited by the outer rim of excitation beam are quenched by the depletion beam by the process of stimulated emission depletion. Due to this, only narrow central portion gives fluorescence thereby decreasing the size of effective collection spot (see Fig. IA), which is equivalent to making a blunt tip of a pen sharper so that it can write sharp letter. To calculate the resolution of STED microscope, the equation (1) has to be modified as (Hell and Wichmann 1994, Hell 2007, Neupane et al. 2013, Neupane et al. 2014, Neupane et al. 2015):

$$
D_{\text {sted }}=\frac{\lambda}{2 N A \sqrt{1+\frac{I}{I_{s}}}}
$$

Where, $\mathrm{I}$ is intensity of depletion laser beam and $\mathrm{I}_{\mathrm{s}}$ is the intensity at which fluorescence intensity decreases to half of its original intensity. I depends on the properties of fluorophore like depletion cross section and fluorescence life time. Equation (3) shows that resolution of STED microscope scales by a factor of $\sqrt{1+1 / t}$. It means STED resolution can provide infinite theoretical resolution. In experiment, the value of the factor in the range of $3-10$ can be obtained resulting in resolution improvement by a factor of 3-10. Resolution of STED microscope is largely affected by the quality of donut beam i.e. intensity profile close to the centre (should be perfectly zero), size of donut, and light polarization (Hell and Wichmann 1994, Neupane et al. 2013)]. Several versions of STED microscope are reported in literature and one of such versions of the microscope assembled by author of this paper at North Carolina State University, USA is shown in figure 1B. Major cost of STED microscope comes from laser and can go as high as $\$ 1$ million. However, using cheaper laser systems the microscope shown in figure $1 \mathrm{~B}$ was assembled roughly at cost of $\$ 0.2$ million.

STED microscopy has great potentialities and in near future it may even find application in early diagnostics of cancer. In recent years, applications of STED microscope in imaging many nanoscopic features in both fixed [healthy cells killed by chemical agents (formalin, butyraldehyde etc.) and stained with dye] and living cells, studying functions of neuronal tissues and cells, and studying movement of biomolecules in living cells are demonstrated. The details, of which can be found in the following works (Hein et al. 2008, Westphal et al. 2008, Leung and Chou 2011, Tonnesen et al. 
2011, Stender et al. 2013, Neupane et al. 2014, Neupane et al. 2015). Up to this point, very few research articles related to applicability of STED microscope in materials science research are published (Harke et al. 2008, Lauterbach et al. 2010).

In this paper, use of the microscope in resolving $200 \mathrm{~nm}$ dye doped polymeric nanoparticle in alumina membrane template is demonstrated. It is found that these particles form linear to quasi polygonal shaped self assembled structures with inter-particle spacing (edge-edge distance) greater than particle diameter. The self assembly is most likely guided by pore pattern in the template and long range interaction forces. The pore periodicity of alumina membrane used in this study is smaller than wavelength of light. So, it is normally expected that in such surface STED may not give good resolution. The observation of good resolution in such surface demonstrates that STED microscopy works fine even on a scattering surface.

\section{Materials and Methods}

\subsection{Optical setup}

A simple schematic of continuous wave STED microscope used in this study is depicted in Fig. 1B [figure reprinted with permission from this author's original paper (Neupane et al. 2013)]. The laser light needed for fluorescence excitation of dye was provided from air cooled Ar ion laser that emits wavelength of $488 \mathrm{~nm}$ (35-LAP-431-240, CVI/Melles Griot). The laser light for stimulated emission depletion of the dye was provided from a fiber diode laser that can give maximum power output of $1000 \mathrm{~mW}$ and wave length of $592 \mathrm{~nm}$ (MPB communication, VFLP-1000-592-OEM1). The excitation and depletion laser lights were made circularly polarized by using a quarter wave plate QWP (CVI/Melles Griot, ACWP-400700-06-4). The spot size of laser in focal plane of microscope objective (Nikon, Plan Apo, 100× /1.40-0.7, Oil) depends on size of laser on the back aperture. To get smallest possible spot in focal plane, the beam was expanded and collimated by a telescope build in $4 \mathrm{f} \mathrm{(} \mathrm{f}=$ focal length) configuration using a pair of plano convex lenses. The donut shaped depletion beam was obtained by sending the laser line through a phase plate (RPC photonics, VPPia) that produces phase ramp of $0-2 \pi$. To get donut having perfectly dark centre the polarization of laser should be very pure. So, before making the light circularly polarized and sending through the vortex phase plate, the laser was cleaned by sending it though a Glantype polarizer (Thorlabs). The both laser lines were routed to the back aperture of the objective lens by using $505 \mathrm{~nm}$ long pass dichroic filter (that reflects/transmits wavelength shorter/longer than $505 \mathrm{~nm}$ ) and $570 \mathrm{~nm}$ dichroic short pass filter (that reflects/transmits wavelength longer/shorter than $570 \mathrm{~nm}$ ). The green fluorescent signal emitted from a sample (that travels in direction opposite to excitation and depletion laser lights) passes through the two dichroic filters. The depletion laser leaking from 570 dichroic was filtered by using sharp edge notch filter (Semrock, NF03-594-E) that blocks background laser light in the range of 584-604 $\mathrm{nm}$ with around 99\% efficiency. The fluorescence signal was sent through a band pass filter that can transmit light in the range of $510-560 \mathrm{~nm}$ with $99 \%$ efficiency. The fluorescent signal having wavelength in the range of $510-560 \mathrm{~nm}$ was sent into a multimode fiber optics (such fiber can send light having all possible transverse mode profiles) pinhole having hole diameter of $50 \mu \mathrm{m}$ (Thorlabs). The other end of fiber optics was connected to a avalanche photodiode detector (APD) that can amplify signal upto 108 times (Perkin Elmer, SPCMAQRH-15-FC). APD is a device that converts very weak light signal to voltage with high quantum yield. The voltage signal, which is directly proportional to amount of light incident in active area of detector was counted by a photon counting
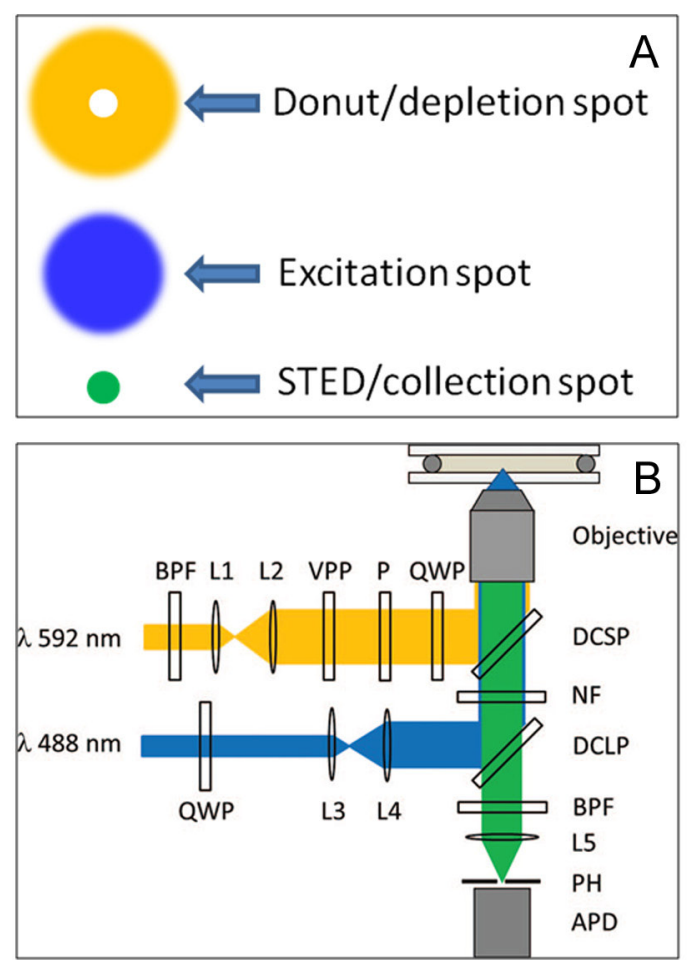

Fig. 1: (A) A simple schematic of depletion laser light (orange), excitation light (blue), and fluorescence collection spot (green) profiles that are observed in focal plane of STED microscope. (B) A simple schematic of continuous wave STED microscope. The light needed for the excitation of green dye is provided by a blue laser line (with wavelength of $488 \mathrm{~nm}$ ) from a air cooled Ar ion laser and light needed for the stimulated emission depletion was provided from a fibre diode laser (with wavelength of 592 $\mathrm{nm})$. In the figure VPP stands for vortex phase plate, $\operatorname{Li}(i=1,2,3,4,5)$ stands for plano convex lens, QWP quarterwave plate, DCSP dichroic mirror short pass, DCLP dichroic mirror long pass, BPF bandpass filter, $\mathrm{P}$ polarizer, NF notch filter, APD avalanche photodiode detector, and $\mathrm{PH}$ pinhole [reprinted with permission from Ref.(Neupane et al. 2013)]. (C)Absorption (black) and emission (blue) spectra of FITC doped polystyrene particles. 

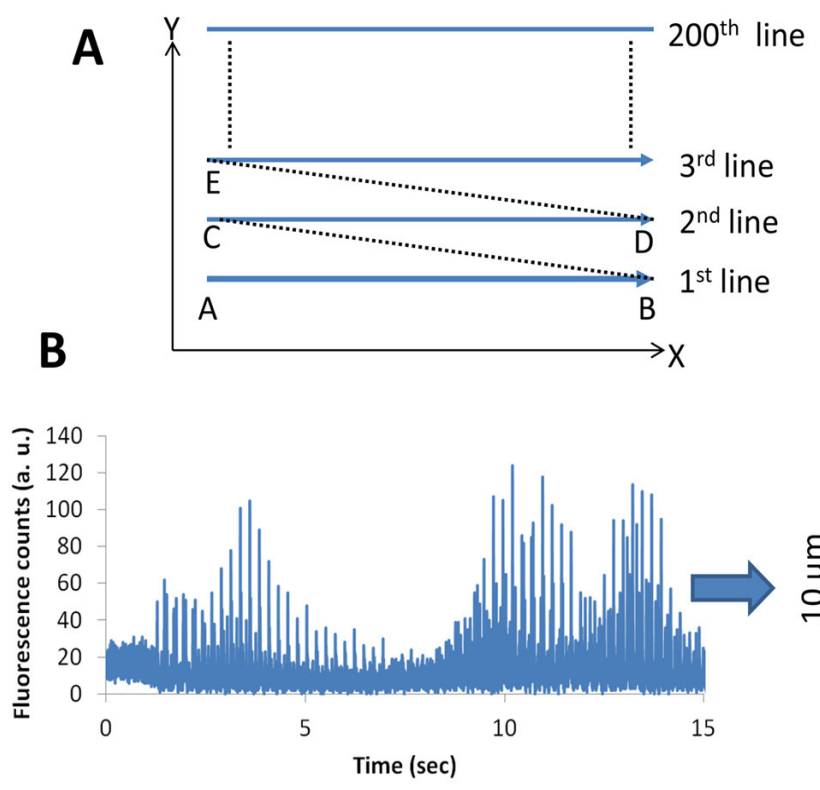

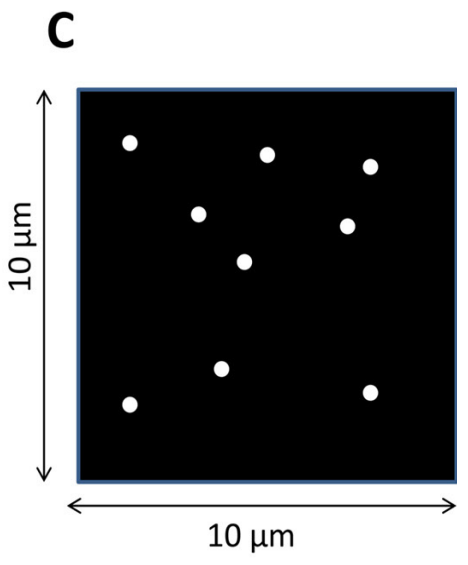

Fig. 2: (A) Imaging scheme used in sample scanning type STED microscope. (B) A representative intensity pattern obtained after scanning. (C) A cartoon image that can be reconstructed if intensity pattern similar to B is used. board controlled by a vendor supplied software. Sample was scanned in XY directions by a piezo-stage (stage coupled to a piezo material that expands or retracts depending on the amount of voltage supplied) that was mounted on a translational stage (PI Nano, Physik Instrumente, P-545). The moving precision of the piezo state was very good $( \pm 1 \mathrm{~nm})$ and scanning range in XYZ direction was $200 \mu \mathrm{m}$. To get sufficient signal to noise ratio, each data point was integrated for 1.0 $\mathrm{ms}$ and acquired data were converted to image by the help of home written plug in program in NIH ImageJ program.

In the figure VPP stands for vortex phase plate, $\mathrm{Li}(\mathrm{i}=1,2,3,4,5)$ stands for plano convex lens, QWP quarterwave plate, DCSP dichroic mirror short pass, DCLP dichroic mirror long pass, BPF bandpass filter, $P$ polarizer, NF notch filter, APD avalanche photodiode detector, and $\mathrm{PH}$ pinhole [reprinted with permission from Ref.(Neupane et al. 2013)]. (C)Absorption (black) and emission (blue) spectra of FITC doped polystyrene particles.

\subsection{Sample preparation}

The carboxylated polystyrene nanoparticles that were doped with fluorescein isothocyanate (FITC) was purchased from from Thermo/Duke Scientific. The size of particles was measured from SEM micrograph and was found to be $190 \pm 10$ $\mathrm{nm}$. The absorption and fluorescence spectra of aqueous suspension of the particle are shown in figure $1 \mathrm{C}$. The absorption and emission peaks appeared around 505 and 515 $\mathrm{nm}$, respectively. The number of particles in original aqueous solution was $\sim 3 \times 10^{18}$ particle $/ \mathrm{mL}$. For self assembly, the solution was diluted 20 times using 18.2-M $\Omega$ Milli-Q water. The anodic membrane (porous alumina membrane) having pore size of $100 \mathrm{~nm}$ and thickness $60 \mu \mathrm{m}$ were purchased from Whatman International (Maidstone, U.K.). Before exposing the membrane with particle suspension the membranes were soaked for 1 hour in 18.2-M $\Omega$ Milli-Q water. After that around $500 \mu \mathrm{L}$ of suspension was added on the top of membrane with membrane resting on a pre- cleaned glass slide. Around 10 $\mu \mathrm{L}$ of 1 molar $\mathrm{NaCl}$ solution was added in the suspension and the membrane incubated for 2 hours at room temperature ( 25
${ }^{\circ} \mathrm{C}$ ). The role of salt was to minimize the surface charge on the nanoparticle (which were originally negatively charged) that assist in self assembly. After the incubation, the membrane was gently washed two times with 18.2-M $\Omega$ Milli-Q water so as to remove moving nanoparticles. Removal of moving particle is important as such particle give unnecessary background during imaging. Right before imaging, a microscope cover slip (Corning, 1.5, 22×22 mm) was glued on the top of membrane in a wet chamber and imaged using the STED microscope.

\section{Results and Discussion}

\subsection{Image formation in sample scanning type STED microscopy}

Image in STED microscope can be acquired either by moving a sample keeping laser beam fixed (also called sample scanning type STED) or by scanning beam by keeping sample fixed (also called beam scanning STED). Scanning is achieved by a scanner-a device that can move very fine steps with precision of $\pm 1 \mathrm{~nm}$. STED is point scanning microscopy in which scanner is moved one step and collected one pixel (one data point), moved to next step and collected next data point, and after collecting many data points image is reconstructed. This imaging scheme is very different from normal photography where many pixels or whole image in collected in single click. A simple scanning scheme used in sample scanning type STED is shown in Fig. 2A. Let us assume scanner is at point $A$ of line $A B$. In moving from $A$ to $B$ along the line $A B$, scanner makes 200 steps with length of one step equal to $50 \mathrm{~nm}$ and time required for one step movement of 1 millisecond. At each step the detector collects one data point. It means that while moving from $A$ to $B, 200$ data points are collected. Now, scanner is moved from $B$ to $C$ at very high speed (around 1000 times higher speed than A to $B$ movement) so that detector cannot collect any data point. Now $C$ to D movements, like A to B movement, is performed and 200 data points are collected. Such scanning is repeated to 200 lines and in total $200 \times 200$ data points (total movement time $=200 \times 200 \mathrm{~ms}=40 \mathrm{~s}$ ) are collected. A typical intensity 

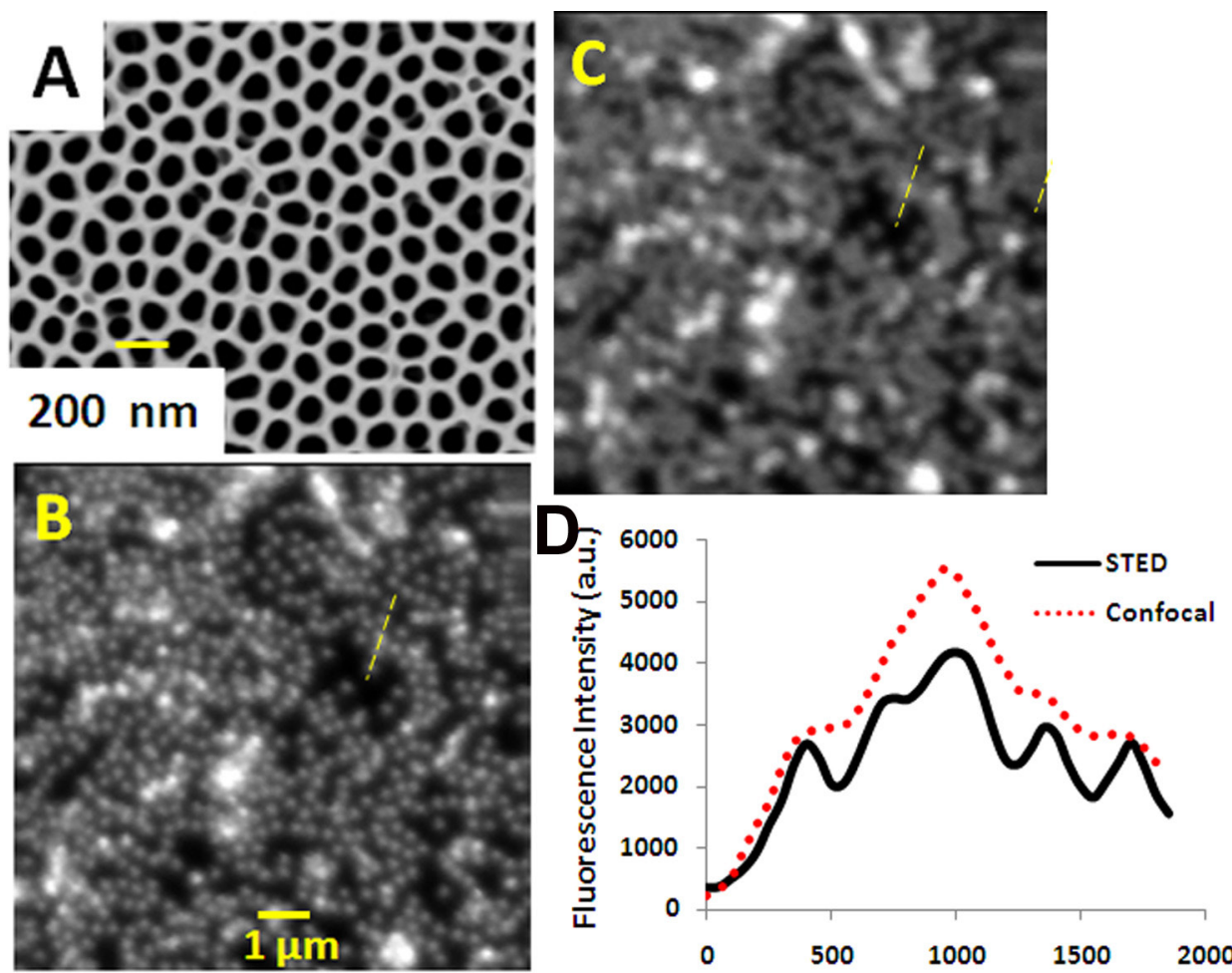

Fig. 3: (A) SEM image of porous alumina membrane used in this study. Scale bar $200 \mathrm{~nm}$. (B) STED image of $200 \mathrm{~nm}$ dye doped nanoparticles self assembled in membrane in wet condition. (C) Confocal microscopic image of same sample. Scale bar shown in B is $1 \mu \mathrm{m}$ and also applies for C. (D) Intensity line profile of particles shown by dotted lines in Figs. B and C.

pattern (intensity as function of scanner movement time) obtained by scanning is shown in figure $2 \mathrm{~B}$. To get image from such data, the intensity data points are folded at every interval of 200 millisecond (time required to move one line) by Matlab written plugin file using ImageJ software. After folding, the data points are converted to image as shown in Fig. 2 C. Since step size in $X$ and $Y$ direction is $50 \mathrm{~nm}$ and total steps are 200 , the $-X Y$ size of image will be $10000 \times 10000$ $\mathrm{nm}(=10 \times 10 \mu \mathrm{m})$. If step size is made $25 \mathrm{~nm}$ the size of image becomes $5 \times 5 \mu \mathrm{m}$ but total time needed to acquire image becomes double (i.e. 80 seconds).

The advantage of point-by-point scanning (over regular photography) is that we can acquire high resolution three dimensional image (3D) of a thick sample like cell and tissues. For that, XY planes or slices are collected at various depths from the surface of sample (i.e. $Z$ direction), then we can reconstruct $3 \mathrm{D}$ image by overlapping these planes. STED can image $3 \mathrm{D}$ biological samples in natural form, which is the biggest advantage over other forms of microscopy that are discussed in introduction section.

\subsection{Self assembly of nanoparticles in alumina membrane in wet condition}

Nanoparticle self assembly is an active area of research as self assembled nano structures are finding applications in many areas such as in electronics, catalysis, surface enhanced Raman spectroscopy, microfluidic detection, template in synthesis of nanostructures, photonic crystal wave guides, etc. Understanding the self assembly pattern in wet and dry condition is very important in finding new applications and also optimizing existing applications (Velev and Gupta 2009, Zhu et al. 2009, Verre et al. 2012).

In this study, the performance of the STED microscope is demonstrated by imaging $200 \mathrm{~nm}$ dye doped nanoparticles, self assembled in anodic membrane surface. Anodic membrane (also called alumina membrane) used in this study shows pores of $100 \mathrm{~nm}$ diameter with well defined periodicity. The SEM image is shown in figure $3 \mathrm{~A}$. Anodic membranes are used in many applications ranging from size selective separation to synthesis of nanomaterials (Yanagishita et al. 2005, Zhao et al. 2006). The STED image of particles self assembled in such membrane is shown in Fig. 3B. For comparison, image of same area (same field of view) measured with low resolution confocal microscope is shown in Fig. $3 \mathrm{C}$. The line profile of few particles (indicated by dotted line in images $3 \mathrm{~B}$ and $3 \mathrm{C}$ ) is shown in figure $3 \mathrm{D}$. The profile shows that STED nicely resolved particles that were unresolved in confocal microscope. Comparison of $3 \mathrm{~B}$ and $3 \mathrm{C}$ images shows that, STED microscope resolves patterns of self assembled nanostructures. It is interesting to note in image $3 \mathrm{~B}$ that nanoparticles form loosely packed short linear chains and some quasi polygonal shapes. The dried self assembly of polymeric nanoparticles is reported to form tightly packed (edge to edge distance smaller than particle diameter) hexagonal structures (Denkov et al. 1992, Dimitrov and Nagayama 1996). It is possible that formation of loose and heterogeneous patterns is due to combined effect of pore periodicity and long range repulsive forces, which stop nanoparticles to come in close contact.

\section{Conclusions}

In this paper a detailed description of Stimulated Emission Depletion (STED) along with comparison with other forms of microscopy is provided. Image collection scheme is also discussed. Application of the microscope in resolving self assembly of $200 \mathrm{~nm}$ particles in alumina membrane is demonstrated. The observation of nicely resolved self assembled pattern on the membrane template proves that STED works fine even on a scattering surface. As compared to self assembly in dried condition, nanoparticles form loosely packed linear and polygonal structures. Formation of such unique pattern may be due to effect of pore periodicity and existence of long range forces.

AcKNowledgements Author acknowledges Kathmandu Institute of Applied Sciences for providing start up funding to write this paper. Author also provides special thanks to Prof. Dr. Gufeng Wang at 
North Carolina State University for giving permission to include few images collected by the author while author was working under him.

\section{References}

Denkov, N., O. Velev, P. Kralchevski, I. Ivanov, H. Yoshimura, and K. Nagayama. 1992. Mechanism of formation of two-dimensional crystals from latex particles on substrates. Langmuir 8:31833190.

Dimitrov, A. S., and K. Nagayama. 1996. Continuous Convective Assembling of Fine Particles into Two-Dimensional Arrays on Solid Surfaces. Langmuir 12:1303-1311.

Harke, B., C. K. Ullal, J. Keller, and S. W. Hell. 2008. Threedimensional nanoscopy of colloidal crystals. Nano Letters 8:1309-1313.

Hein, B., K. I. Willig, and S. W. Hell. 2008. Stimulated emission depletion (STED) nanoscopy of a fluorescent protein-labeled organelle inside a living cell. Proceedings of the National Academy of Sciences of the United States of America 105:1427114276.

Hell, S. W. 2007. Far-Field Optical Nanoscopy. Science 316:1153-1158.

Hell, S. W., and J. Wichmann. 1994. Breaking the diffraction resolution limit by stimulated emission: stimulated-emissiondepletion fluorescence microscopy. Opt. Lett. 19:780-782.

Lauterbach, M. A., C. K. Ullal, V. Westphal, and S. W. Hell. 2010. Dynamic Imaging of Colloidal-Crystal Nanostructures at 200 Frames per Second. Langmuir 26:14400-14404.

Leung, B. O., and K. C. Chou. 2011. Review of Super-Resolution Fluorescence Microscopy for Biology. Applied Spectroscopy 65:967-980.

Müller, M. 2006. Introduction to confocal fluorescence microscopy. SPIE PRESS, Washington.

Neupane, B., F. Chen, W. Sun, D. T. Chiu, and G. Wang. 2013. Tuning donut profile for spatial resolution in stimulated emission depletion microscopy. The Review of Scientific Instruments 84:043701.
Neupane, B., T. Jin, L. Mellor, E. Loboa, F. Ligler, and G. Wang. 2015. Continuous-Wave Stimulated Emission Depletion Microscope for Imaging Actin Cytoskeleton in Fixed and Live Cells. Sensors 15:24178.

Neupane, B., F. S. Ligler, and G. Wang. 2014. Review of recent developments in stimulated emission depletion microscopy: applications on cell imaging. Journal of Biomedical Optics 19:080901-080901.

Stender, A. S., K. Marchuk, C. Liu, S. Sander, M. W. Meyer, E. A. Smith, B. Neupane, G. Wang, J. Li, J.-X. Cheng, B. Huang, and N. Fang. 2013. Single Cell Optical Imaging and Spectroscopy. Chemical Reviews 113:2469-2527.

Tonnesen, J., F. Nadrigny, K. I. Willig, R. Wedlich-Soldner, and U. V. Nagerl. 2011. Two-Color STED Microscopy of Living Synapses Using A Single Laser-Beam Pair. Biophysical Journal 101:25452552.

Velev, O. D., and S. Gupta. 2009. Materials Fabricated by Micro- and Nanoparticle Assembly - The Challenging Path from Science to Engineering. Advanced Materials 21:1897-1905.

Verre, R., K. Fleischer, O. Ualibek, and I. V. Shvets. 2012. Selfassembled broadband plasmonic nanoparticle arrays for sensing applications. Applied Physics Letters 100.

Westphal, V., S. O. Rizzoli, M. A. Lauterbach, D. Kamin, R. Jahn, and S. W. Hell. 2008. Video-rate far-field optical nanoscopy dissects synaptic vesicle movement. Science 320:246-249.

Yanagishita, T., K. Nishio, and H. Masuda. 2005. Fabrication of Metal Nanohole Arrays with High Aspect Ratios Using TwoStep Replication of Anodic Porous Alumina. Advanced Materials 17:2241-2243.

Zhao, L., M. Yosef, M. Steinhart, P. Göring, H. Hofmeister, U. Gösele, and S. Schlecht. 2006. Porous Silicon and Alumina as Chemically Reactive Templates for the Synthesis of Tubes and Wires of $\mathrm{SnSe}, \mathrm{Sn}$, and $\mathrm{SnO} 2$. Angewandte Chemie International Edition 45:311-315.

Zhu, K., D. G. Wang, and J. Liu. 2009. Self-Assembled Materials for Catalysis. Nano Research 2:1-29. 